Revista em Agronegócio e Meio Ambiente, Maringá (PR)

DOI: http://dx.doi.org/10.17765/2176-9168.2018v11n2p561-572

\title{
ADUBO ORGÂNICO CONSORCIADO COM GRAMÍNEAS PARA O CULTIVO DO COGUMELO Ganoderma lucidum
}

\author{
André Luiz Merthan Saad* \\ Fernanda Silva Lima** \\ Meire Cristina Nogueira de Andrade***
}

RESUMO: O objetivo do presente trabalho foi avaliar o uso de um adubo orgânico com dois tipos de gramíneas, capim Coast-cross (Cynodon dactylon (L.) Pers) e capim alfafa (Medicago sativa L.) em comparação ao substrato tradicional enriquecido com o farelo de trigo. Foram utilizadas duas linhagens do Ganoderma lucidum, 144 e 351, esperando identificar a melhor opção de substrato de cultivo, tendo como critérios de avaliação a composição química dos substratos, a eficiência biológica, além da quantificação dos basidiomas frescos e o número de basidiomas. Foram testados quatro substratos, sendo S-1 (alfafa + farelo), S-2 (alfafa + adubo orgânico), S-3 (Coast-cross + farelo), S-4 (Coast-cross + adubo orgânico), cada qual com duas linhagens do G. lucidum. O experimento foi composto por dez repetições, totalizando 80 pacotes de $700 \mathrm{~g}$ de substrato cada. Aos tratamentos adicionaram-se $80 \%$ de capim, $18 \%$ de farelo de trigo ou adubo orgânico e $2 \%$ calcário calcítico, com umidade ajustada para $60 \%$. As melhores médias de $\mathrm{EB}, \mathrm{MBF}$ e NB demonstraram que os substratos que melhor apresentaram resultados agronômicos foram os substratos S2 (Alfafa + adubo) e o substrato S4 (Coast-cross + Adubo). A linhagem 144 do G. lucidum, foi a que apresentou melhor resultado e desempenhos de produtividade.

PALAVRAS-CHAVE: Capins; Substratos; Fungos.

\section{CONSORCED ORGANIC FERTILIZER WITH GRASSES FOR GROWING MUSHROOM Ganoderma lucidum}

ABSTRACT: The use of organic fertilizer with two types of grass, coast-cross ( $C y$ nodon dactylon (L.) Pers) and lucerne/alfafa grass (Medicago sativa L.), was analyzed and compared to traditional substrate enriched with wheat bran. Two strains of Ganoderma lucidum, 144 and 351, were used to identify the best option for

\footnotetext{
Mestrando em Agronomia pela Faculdade de Ciências Agronômicas de Botucatu - FCA/UNESP, Brasil

** Mestre em Ciência e Tecnologia Ambiental pela Universidade do Sagrado Coração - USC, Brasil.

*** Doutora em Agronomia, Docente permanente do Programa de Pós-graduação em Agronomia (Energia na Agricultura) da Faculdade de Ciências Agronômicas de Botucatu - FCA/UNESP, Brasil.

E-mail: mcnandrade@hotmail.com
} 
the substrate. Evaluation criteria comprised the chemical composition of the substrates, biological efficiency and the amount of fresh basidioms and the number of basidioms. Four substrates were tested, namely, S-1 (alfafa + bran), S-2 (alfafa + organic fertilizer), S-3 (coast-cross + bran), S-4 (coast-cross + organic fertilizer), with the two $G$. lucidum strains. Experiment comprised ten replications, with 80 packages with $700 \mathrm{~g}$ of substrate each. Treatments also received $80 \%$ grass, $18 \%$ wheat bran or organic fertilizer and $2 \%$ limestone, with $60 \%$ humidity. Best means for EB, MBF and NB showed that S2 (alfafa + fertilizer) and S4 (coast-cross + fertilizer) were the substrates with the best agronomic results. Strain 144 of G. lucidum had the best result and yield in productivity.

KEY WORDS: Grass; Substrates; Mushrooms.

\section{INTRODUÇÃO}

A seleção adequada do substrato é de fundamental importância para o sucesso de qualquer tipo de produção de cogumelos. Os resíduos de origem agroindustrial, tais como capins, polpa de café, farelo de cereais, bagaço de cana, cascas de frutas processadas, batata, farinha de cereais, mandioca, entre outros são substratos bastante difundidos nesses processos (ALQUATI et al., 2016; CARVALHO et al., 2015; GURUNG et al., 2012; LÓPEZ et al., 2011; ERKEL, 2009).

Os fungos do gênero Ganoderma são conhecidos popularmente como orelha-de-pau pelos chineses e Ling Zi para os japoneses. Diante das suas inúmeras propriedades, são relatados principalmente pelo seu poder medicinal (LIN, 2009).

Originalmente, na China, seu cultivo era feito em toras e serragens de espécies arbóreas (Pinus spp., Eucalyptus spp., entre outras). Este tipo de substrato foi substituído gradativamente por gramíneas diversas, por razões de ordem ecológica e preservação das espécies nativas e exóticas (LIN, 2009). Somam-se a isso o fato de a conversão de energia solar em espécies de gramíneas ser seis a oito vezes maior que a de espécies de arbóreas utilizadas no cultivo tradicional. Os fungos cultivados em gramíneas têm maior produtividade que os cultivados em serragem, podendo ser até 30\% superior, e com igual ou maior qualidade nutricional (ZHANXI; ZHANHUA, 2001).

O cogumelo G. lucidum é classificado como lignocelulolítico, pois se desenvolve naturalmente em substratos ricos em lignina e celulose, degradando esses 
compostos (ERKEL, 2009). Consequentemente, tem afinidade por grande variedade de resíduos, incluindo as gramíneas. As vantagens mencionadas fazem com que o cultivo de G. lucidum atraia cada vez mais o interesse de investidores e produtores rurais, como alternativa para complementar a renda familiar, além de se configurarem em uma possibilidade ecologicamente interessante para o aproveitamento de resíduos. No entanto, no Brasil, o G. lucidum é ainda pouco cultivado e o substrato mais utilizado é à base de serragem de eucalipto.

As gramíneas utilizadas em pastagens são substratos potenciais e de qualidade elevada para o cultivo de cogumelos. No entanto, há a carência de pesquisas relacionadas à utilização proporcional e adequada das gramíneas para a formulação do substrato. Há necessidade de se comparar a resposta de várias linhagens do cogumelo em vários tipos de palhas em termos de produtividade e qualidade dos cogumelos obtidos. Condições de clima e de grau de tecnologia dos cultivos também devem fazer parte destes estudos.

A substituição de substratos tradicionais à base de madeira (toras e serragem) por capins, palhas de cereais e outros resíduos agrícolas pode dispensar a suplementação nitrogenada, além da redução no custo de produção. Uma investigação feita por Zhanxi e Zhanhua (2001) revelou que alguns capins podem substituir a serragem e, parcialmente, o farelo arroz no cultivo de Pleurotus ostreatus. Dias et al., (2003) relatam que a palha de feijão para o cultivo de $P$. sajor-caju dispensa suplementação nitrogenada. Além de produtividade elevada e fácil adaptação no Brasil, os conteúdos de proteínas, nitrogênio, gordura, fósforo, potássio e magnésio, em gramíneas são maiores do que aqueles em serragem (ZHANXI; ZHANHUA, 2001).

Assim, objetivou-se na presente pesquisa analisar a viabilidade do uso de um adubo orgânico ${ }^{4}$ em comparação ao farelo de trigo tradicional consorciado com dois tipos de gramíneas: capim coast-cross (Cynodon dactylon (L.) Pers) e capim alfafa (Medicago sativa L.) nas linhagens 144 e 351 de G. lucidum.

\section{MATERIAL E MÉTODOS}

O experimento foi conduzido em duas etapas: 1- preparo do Spawn (inócu-

\footnotetext{
$\overline{4}$ O adubo orgânico foi fornecido pela empresa Sobrafértil de Bauru, SP, por meio de uma parceria já estabelecida entre a empresa citada e a USC. Tal composto é produzido utilizando como matéria-prima sobra de alimentos recolhidos em hotéis e restaurantes locais.
} 
lo do fungo de interesse, neste caso, o micélio de G. lucidum cultivado em substrato) e dos substratos, inoculação e incubação na empresa Yuki cogumelos, na cidade de Araçoiaba da Serra, SP; 2- produção e colheita na Estância Saad, localizada na cidade de Botucatu, SP.

\subsection{PREPARO DO SPAWN}

Esta fase da pesquisa consistiu no preparo do Spawn para propagação das linhagens 144 e 351 de G. lucidum, sendo que ambas as linhagens foram obtidas da Embrapa - Recursos Genéticos e Biotecnologia - Cenargem, Brasília, DF. Nesta etapa foi feito o cozimento das sementes de trigo em água fervente (na proporção 1 $\mathrm{kg} / 2$ L semente/água) durante 40 minutos. Logo depois do seu resfriamento, foram adicionados $2 \%$ de calcário calcítico (em relação ao peso seco das sementes) e misturados em uma betoneira de uso na construção civil, para facilitar a homogeneização.

Em seguida, as sementes foram acondicionadas em pacotes de PEAD (Polietileno de Alta Densidade) e esterilizadas em autoclave a uma temperatura de $121^{\circ} \mathrm{C}$ por um período de 4 horas.

Depois de esterilizados e com temperatura ambiente dos pacotes, os mesmos foram transportados até um laboratório com câmara de fluxo laminar, para serem inoculados com o Spawn do G. lucidum, e permaneceram armazenados em temperatura controlada de $25^{\circ} \mathrm{C}$, por duas semanas, até atingirem o ponto ideal (colonização total dos grãos - Spawn) para serem inoculados nos tratamentos com o adubo orgânico e o farelo.

\subsection{PREPARO DOS SUBSTRATOS}

Os capins Coast-cross (Cynodon dactylon (L.) Pers) e alfafa (Medicago sativa L.) foram comprados em uma loja agropecuária na cidade de Botucatu, SP. O adubo orgânico foi fornecido pela empresa Sobrafertil, localizada na cidade de Bauru, SP, por meio de uma parceria com a Universidade do Sagrado Coração, localizada também na cidade de Bauru. $\mathrm{O}$ adubo produzido por um processo de compostagem, utilizando como matéria-prima sobra de alimentos recolhidos de hotéis e restaurantes locais. 
Depois da obtenção dos capins e o adubo orgânico, os mesmos foram encaminhados para a Empresa Yuki Cogumelos, na cidade de Araçoiaba da Serra, onde passaram por um processo de moagem por um triturador convencional para atingir a granulometria necessária $(1-5 \mathrm{~mm})$ para o desenvolvimento do fungo. $\mathrm{O}$ material triturado foi pesado para ser distribuído nos pacotes de PEAD, antes de passar para o processo de esterilização. As formulações dos tratamentos experimentais (composição dos substratos) seguiram o mesmo procedimento para todos os pacotes, sendo compostas (em peso seco) por $80 \%$ de capim, $2 \%$ de calcário calcítico, $18 \%$ de farelo de trigo ou adubo orgânico. A essas misturas foram acrescidos $60 \%$ de água na sua composição.

A seguir, o material foi homogeneizado em uma betoneira de uso na construção civil e prensado. Cada pacote recebeu um respiro, em sua parte superior para permitir a troca gasosa e o desenvolvimento do G. lucidum. Todos os pacotes foram submetidos ao processo de esterilização a $121^{\circ} \mathrm{C}$, durante 4 horas, para que somente o fungo desejado fosse desenvolvido na produção, evitando assim a contaminação dos pacotes.

A inoculação dos pacotes com a linhagem G. lucidum foi feita após o resfriamento dos pacotes até a temperatura ambiente, em laboratório, utilizando uma câmera de fluxo laminar em condições assépticas adequadas, evitando a contaminação por outros microrganismos.

Após este procedimento, os mesmos foram levados a uma sala climatizada e mantidos a uma temperatura de $25^{\circ} \mathrm{C}$ até a completa colonização dos substratos, que perdurou por aproximadamente por duas semanas.

O delineamento experimental foi inteiramente casualizado, em esquema fatorial $4 \times 2$, correspondente a quatro tipos de substratos $(S 1=$ Alfafa + farelo; S2 = alfafa + adubo; $S 3=$ capim coast-cross + farelo e; $S 4=$ capim coast-cross + adubo) e duas linhagens de G. lucidum (144 e 351), cada qual com dez repetições (bloco de substrato de $700 \mathrm{~g}$ ), totalizando 80 unidades experimentais.

\subsection{PRODUÇÃO E COLHEITA}

Depois de colonizados pelo micélio de G. lucidum, os pacotes foram transferidos para uma estufa rústica, feita com estrutura de bambu e coberta com plásti- 
co transparente com espessura de 150 micras, instalada em uma propriedade rural denominada Estância Saad, na cidade de Botucatu, SP, com clima tropical, média de temperatura entre 23 e $32{ }^{\circ} \mathrm{C}$ no verão e 18 a $23^{\circ} \mathrm{C}$ no inverno. A referida propriedade possui as seguintes coordenadas geográficas: latitude $23^{\circ} 01^{\prime} 44.0$ Sul e longitude $48^{\circ} 29 ' 16.8$ Oeste.

Os pacotes foram colocados na estufa, sendo distribuídos em ordem por tratamento, para facilitar na hora da colheita. Neste momento foram cortadas as partes de cima dos pacotes, deixando o substrato com os cogumelos a mostra, favorecendo maior ventilação.

A temperatura média na estufa foi mantida em $25 \pm 5^{\circ} \mathrm{C}$ e umidade relativa de $60-85 \%$, sendo necessário molhar constantemente sempre que o piso de terra ficasse seco. Depois de 30 dias os cogumelos começaram a se desenvolver.

Após 60 dias, observou-se a cor dos cogumelos pelas suas diferentes tonalidades e texturas para quantificar a maturação e o desenvolvimento a serem utilizados como parâmetros na decisão de colheita. A colheita dos basidiomas ocorreu após 90 dias, constituindo apenas em torcer e arrancancá-los dos pacotes. Todos os cogumelos colhidos foram pesados com uma balança de precisão e colocados em embalagens plásticas identificadas conforme o tratamento.

Nesta etapa foi quantificado o número de basidiomas apresentados por cada tratamento, bem como as massas dos basidiomas frescos.

A produtividade de cada tratamento foi expressa pela eficiência biológica (EB), massa dos basidiomas frescos (MBF) e número de basidiomas (NB).

O cálculo para obtenção da eficiência biológica está descrito de acordo com a equação 1:

\section{Eficiência biológica}

Massa fresca total de cogumelos (g)

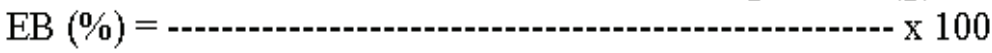

Massa seca do substrato inicial (g) 


\subsection{ANÁLISE ESTATÍSTICA}

Os dados foram obtidos e submetidos à análise de variância e as médias comparadas pelo teste de Tukey (5\%). Para tanto, foi utilizado o programa SISVAR 4.2, desenvolvido pelo Departamento de Ciências Exatas da Universidade Federal de Lavras, Minas Gerais (UFLA).

\section{RESULTADOS E DISCUSSÃO}

Na Tabela 1 encontram-se os valores de F obtidos na análise de variância dos dados de Eficiência biológica (EB), massa dos basidiomas frescos (MBF) e número de basidiomas (NB) para os tipos de substratos e linhagens do G. lucidum.

Tabela 1. Valores de $\mathrm{F}^{1}$ obtidos pela análise de variância de eficiência biológica (EB), massa fresca dos basidiomas (MBF) e número dos basidiomas (NB) de duas linhagens de Ganoderma lucidum cultivadas em quatro tipos de substratos, durante 90 dias de cultivo

\begin{tabular}{cccc}
\hline Fator de variação & EB & MBF & NB \\
\hline Substrato & $154,1^{* *}$ & $71,9^{* *}$ & $32,6^{* *}$ \\
Linhagem & $14,5^{* *}$ & $41,1^{* *}$ & $96,6^{* *}$ \\
Substrato x Linhagem & $2,0^{\mathrm{NS}}$ & $3,4^{* *}$ & $6,2^{* *}$ \\
\hline
\end{tabular}

**Significativo ao nível de 5\%.

NS Não significativo.

${ }^{1}$ Cada estatística $\mathrm{F}$ é uma proporção de médias quadradas.

Podemos observar que houve um efeito significativo da interação substrato versus linhagem para MBF e NB (Tabela 1). Sendo assim, foi realizado o desdobramento dos dados, fazendo-se uma análise comparativa entre os substratos utilizando cada uma das linhagens e outra comparando as linhagens em cada um dos substratos testados (Tabela 2). 
Tabela 2. Eficiência biológica, massa dos basidiomas frescos, número de basidiomas em relação aos substratos e as linhagens do Ganoderma lucidum

\begin{tabular}{|c|c|c|}
\hline \multirow{2}{*}{ Substrato } & \multicolumn{2}{|c|}{ Linhagens } \\
\hline & 144 & 351 \\
\hline \multicolumn{3}{|c|}{ Eficiência biológica (\%) } \\
\hline S1 & $65,7 \mathrm{Ba}$ & $59,7 \mathrm{Ca}$ \\
\hline S2 & $113,7 \mathrm{Aa}$ & $110,6 \mathrm{Aa}$ \\
\hline S3 & $64,0 \mathrm{Ba}$ & $57,8 \mathrm{Ca}$ \\
\hline S4 & $110,1 \mathrm{Aa}$ & $93,1 \mathrm{Bb}$ \\
\hline \multicolumn{3}{|c|}{ Massa dos basidiomas frescos (g) } \\
\hline S1 & $6,5 \mathrm{Ba}$ & $1,5 \mathrm{Ba}$ \\
\hline S2 & $26,5 \mathrm{Aa}$ & $16,9 \mathrm{Ab}$ \\
\hline S3 & $4,7 \mathrm{Ba}$ & $0,0 \mathrm{Ba}$ \\
\hline S4 & $31,7 \mathrm{Aa}$ & $16,4 \mathrm{Ab}$ \\
\hline \multicolumn{3}{|c|}{ Número dos basidiomas } \\
\hline S1 & $1,9 \mathrm{Ca}$ & $0,4 \mathrm{BCb}$ \\
\hline S2 & 3,6 Ba & $2,3 \mathrm{Ab}$ \\
\hline S3 & $1,7 \mathrm{Ca}$ & $0,0 \mathrm{Cb}$ \\
\hline S4 & $4,8 \mathrm{Aa}$ & $1,3 \mathrm{ABb}$ \\
\hline
\end{tabular}

Legenda: S1: 80\% Alfafa + 18\% Farelo, S2: 80\% Alfafa + 18\% Adubo orgânico, S3: 80\% Coast-cross +18\% Farelo, S4: 80\% Coast-cross + 18\% Adubo orgânico.

Nota: Médias seguidas de letras iguais maiúsculas na coluna, dentro de cada parâmetro de produção (EB, MBF e NB), não diferenciam estatisticamente entre si e; letras minúsculas iguais na linha não diferem estatisticamente entre si. Média de 10 repetições.

A eficiência biológica de cada tratamento é correspondente à conversão da matéria do substrato em biomassa dos cogumelos. Este índice é o mais utilizado pelos pesquisadores, o que facilita a comparação dos resultados com a literatura. Na Tabela 2 é caracterizada a comparação entre as linhagens 144 e 351 e a porcentagem de cada tratamento em relação à eficiência biológica, massa dos basidiomas frescos e número de basidiomas. 
As médias de $\mathrm{EB}, \mathrm{MBF}$ e NB evidenciaram que os substratos que apresentaram resultados agronômicos satisfatórios foram os substratos $\$ 2$ (Alfafa + adubo) e o substrato S4 (Coast-cross + Adubo) (Tabela 2).

Em relação à interação entre as linhagens, foi observado que linhagem 144 foi superior em desempenho em comparação à linhagem 351 (Tabela 2), principalmente em relação à eficiência biológica (EB) e ao número de basidiomas (NB). Foi possível verificar que, dependendo da composição do substrato utilizado, ocorreram variações de eficiência e produtividade entre as linhagens. Resultados similares foram obtidos por Rolin et. al (2014) que, comparando substratos e linhagens de $G$. lucidum, relatam que a linhagem 144 apresentou melhor desempenho do que as demais analisadas.

Os resultados de eficiência biológica na presente pesquisa tiverem seus melhores desempenhos proporcionados pelos S2 (Alfafa + adubo) e o substrato S4 (Coast-cross + adubo), 113,7\% e 110,1\%, respectivamente (Tabela 2). Comparando estes resultados com outros da literatura, verificou-se que estes foram satisfatórios. Gurung et al. (2012), utilizando resíduos de serralheria testando diversas fontes de suplementação, obtiveram seu maior índice de eficiência biológica de 22,62\% utilizando serragem de Alnus nepalensis suplementado com farinha de grão-de-bico, uma árvore do Nepal. Erkel (2009) obteve o seu melhor resultado utilizando substrato à base de serragem de álamo com farelo de trigo, cuja eficiência biológica foi de 20,85\%. Carvalho et al. (2015) obtiveram a melhor eficiência biológica com 6,7\%, utilizando a mistura de palha de feijão suplementado com $20 \%$ de farelo de trigo.

Fazendo uma análise comparativa da caracterização química dos insumos utilizados, observaram-se escassas diferenças em relação à porcentagem de nitrogênio $(\mathrm{N})$, carbono $(\mathrm{C})$, relação $\mathrm{C} / \mathrm{N}$ e umidade (Tabela 3). Segundo Urben et. al (2004), as principais fontes de carbono e, consequentemente, de energia de um substrato vegetal são os polissacarídeos e a lignina, constituintes da parede celular, embora existam outros componentes, como lipídeos e proteínas, que podem ser utilizados como fontes de energia pelo G. lucidum. De acordo com Chang e Miles (1989), os requerimentos nutricionais para o crescimento micelial e para a produção de cogumelos da espécie G. lucidum são diferentes. Esta informação foi fundamental para o desenvolvimento de um substrato utilizando o adubo orgânico, permitindo condições favoráveis para a produção do G. lucidum. 
Tabela 3. Caracterização química dos insumos nitrogenados (adubo orgânico e farelo de trigo)

\begin{tabular}{lcccc}
\hline & $\mathbf{N}, \%$ & $\mathbf{C}, \%$ & $\mathbf{C} / \mathbf{N}$ & Umidade, \% \\
\hline Adubo orgânico & 2,3 & 42 & 18 & 12 \\
Farelo de trigo & 2,7 & 53 & 19,6 & 8 \\
\hline
\end{tabular}

$\mathrm{N}(\%)=$ porcentagem de nitrogênio; $\mathrm{C}(\%)=$ porcentagem de carbono; $\mathrm{C} / \mathrm{N}=$ relação entre carbono e nitrogênio.

A escolha correta do substrato depende dos materiais de fácil acesso ao produtor, região e logística. A energia contida no substrato é proveniente principalmente de materiais nitrogenados e, quanto maior seu nível, mais caro fica o substrato para o cultivo. Por isso devem-se estudar as melhores condições e região para cada linhagem do fungo escolhido para o cultivo.

\section{CONCLUSÕES}

Os melhores resultados agronômicos para a produção de G. lucidum foram proporcionados pelos substratos S2 (Alfafa + adubo) e S4 (Coast-cross + adubo).

A linhagem 144 do G. lucidum foi a que apresentou melhores desempenhos de produtividade.

\section{REFERÊNCIAS}

ALQUATI, G. P.; SIQUEIRA, O. A. P. A.; SAAD, A. L. M.; VIANA, S. R. F.; ANDRADE, M. C. N. Residues from urban vegetable pruning in the production of the medicinal mushroom Ganoderma lucidum. African Journal of Agricultural Research, v. 11, p. 412-418, 2016.

CARVALHO, C. S. M.; SALES-CAMPOS, C.; CARVALHO, L. P.; MINHONI, M. T. A.; SAAD, A. L. M.; ALQUATI, G. P.; ANDRADE, M. C. N. Cultivation and bromatological analysis of the medicinal mushroom Ganoderma lucidum (Curt.: Fr.) P. Karst cultivated in agricultural waste. African Journal of Agricultural Research, v. 14, n. 5, p. 3664-3670, 2015. 
CHANG, S.T.; MILES, P.G. Edible mushrooms and their cultivation. Florida: CRC Press; Inc. Boca Raton, 1989.

DIAS, E.S; KOSHIKUMO, E. M. S.; SCHWAN, R. F.; SILVA, R. Cultivo do cogumelo Pleurotus sajor caju em diferentes resíduos agrícolas. Ciência e Agrotecnologia, v.27, n. 6, p.1363-1369, 2003.

ERKEL, E. I. The effect of different substrate mediums on yield of Ganoderma lucidum (Fr.) Karst. Journal of food, agriculture and environment, Helsinki, v. 7, p 841-844, Oct. 2009. Disponível em: < http://world-food.net/the-effect-of-differentsubstrate-mediums-on-yield-of-ganoderma-lucidum-fr-karst/ $>$. Acesso em: 15 mar. 2015.

GURUNG, O.K.; BUDATHOKI, U.; PARAJULI, G. Effect of different substrates on the production of Ganoderma lucidum (Curt.: Fr.) Karst. Our Nature, v.5, n.10, p. 191$198,2012$.

LIN, Z. B. Lingzhi: From Mystery to Science. Beijing, China: Peking University Medical Press, 2009.

LÓPEZ A. M. T; DÍAZ, J. C. Q.; GARCÉS, L. A. Efecto de nutrientes sobre la producción de biomasa del hongo medicinal Ganoderma lucidum. Revista Colombiana de biotecnologia, Bogotá, v. 13, p. 103-109, 2011. Disponível em:<http://www. revistas.unal.edu.co/index.php/biotecnologia/article/viewFile/22949/23752>. Acesso em: 15 mar. 2015.

ROLIM, L.N.; SALES-CAMPOS, C. CAVALCANTI, M. A. Q.; URBEN, A. F. Application of Chinese Jun-Cao technique for the production of Brazilian Ganoderma lucidum strains. Brazilian Archives of Biology and Technology. Brazilian Archives of Biology and Technology, v. 57, n. 3, p. 367-373. 2014.

URBEN, A.F. Produção de cogumelos por meio de tecnologia chinesa modificada. Brasília: EMBRAPA Recursos Genéticos e Biotecnologia, 2004. 186p.

ZHANXI, L.; ZHANHUA, L. Juncao technology. Beijing: China Agricultural Scientech, 2001. 252p. 
Recebido em: 25/11/2016

Aceito em: 16/06/2017 